\title{
Is liking and intake of brassicaceous vegetables determined by PROP taster status and TAS2R38 genotype?
}

\author{
Y. Shen ${ }^{1 *}$, E. Deaville ${ }^{1}$, O. B. Kennedy ${ }^{1}$ and L. Methven ${ }^{1}$ \\ ${ }^{1}$ Department of Food and Nutritional Sciences, University of Reading, Whiteknights, PO Box 226, Reading, Berks, \\ RG6 6AP, UK
}

Increasing vegetable intake may reduce the risk of cancer and cardiovascular diseases ${ }^{(1)}$, yet vegetable consumption remains low ${ }^{(2)}$. Bitter taste contributes to consumer rejection of vegetables, especially brassicaceous vegetables (BV). Individual taste sensitivity is affected by genotype for the hTAS2R38 bitter taste receptor which determines ability to taste the bitter thiourea group $(\mathrm{N}-\mathrm{C}=\mathrm{S})$ present in both 6-npropylthiouracil (PROP) and in glucosinolate compounds found in $\mathrm{BV}^{(3)}$.

This study investigated the associations between PROP taster status and hTAS2R38 genotype and their influence on vegetable liking, perceived bitterness intensity and intake of BV. A total of 49 volunteers (aged 18-55 years) were recruited and classified into supertaster, medium taster and non-taster groups using perceived intensity ratings for both PROP and sodium chloride solutions. DNA from saliva was hTAS2R38 genotype tested. Liking of raw vegetables (broccoli and white cabbage (BVs), spinach and courgette (non-BVs)) was rated (9-point hedonic scale) and perceived vegetable bitterness (labelled magnitude scale). Participants' diet was assessed using Food Frequency Questionnaire (FFQ).

Using PROP, $41 \%$ of participants were classified as supertasters (ST), $46 \%$ medium tasters (MT) and 13\% non-tasters (NT). However, genotyping designated less participants into the supertaster category, finding $23 \%$ subjects had the two sensitive alleles (PAV/PAV), $40 \%$ had both insensitive alleles (AVI/AVI) and 37\% had PAV/AVI. There was no significant difference in liking of the 4 vegetables overall nor between taster or genotype groups. However, those with supertaster status and PAV/PAV genotype tended to find BV more bitter than non-BV ( $p=0.06$ for genotype*vegetable). Mean bitter rating of BV for the PAV/PAV participants was 3 fold higher than for non-BV, whereas for other participant's ratings between BV/non-BV differed 1.5 fold. FFQ found the proportion of AVI/AVI genotype participants reporting to never/ rarely eat some named BVs was lower than for PAV/PAV participants (comparing AVI/AVI to PAV/PAV for nonconsumers of brussels sprouts $57 \% / 73 \%$; cabbage $7 \% / 45 \%$ and cauliflower $29 \% / 45 \%$ ). A similar trend emerged for taster status groups (comparing NT to ST for non-consumers of brussels sprouts $50 \% / 70 \%$; cabbage $0 \% / 40 \%$ and cauliflower $33 \% / 45 \%$ ).

The preliminary findings of this study suggest that individuals showing increased bitter taste sensitivity (PROP taster status and hTAS2R38 genotype) may have decreased liking and intakes of brassicaceous vegetables.

1. Hung HC et al. (2004) Fruit and Vegetable Intake and Risk of Major Chronic Disease J National Cancer Inst 96(21), $1577-1584$.

2. CabinetOffice (2008) Food Matters. URL:http://webarchive.nationalarchives.gov.uk/+/http:/www.cabinetoffice.gov.uk/media/cabinetoffice/strategy/ assets/food/food matters1.pdf [9 April 2012].

3. Sandell MA \& Breslin PA (2006) Variability in a taste-receptor gene determines whether we taste toxins in food, Current Biology 16(18), R792-94. 